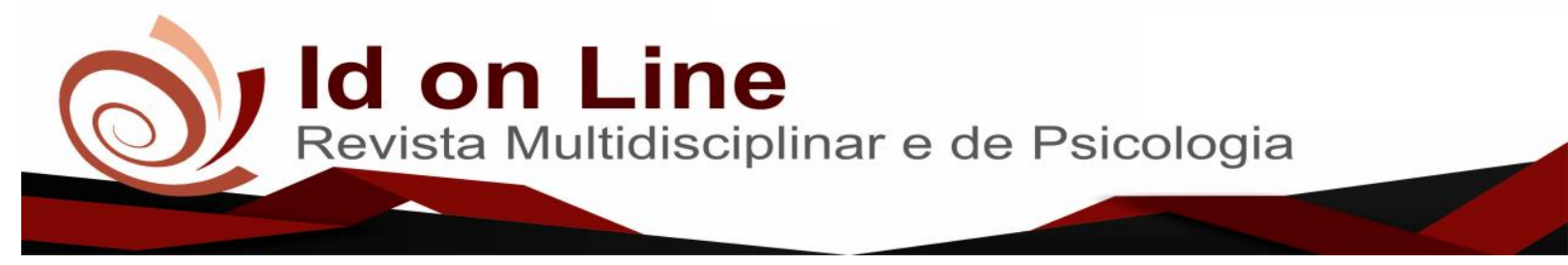

Artigo de Revisão

\title{
A influência do tabagismo na doença periodontal: Uma Revisão de Literatura
}

Graziele Veronica Pereira Medeiros ${ }^{1}$; Karina Sarno Paes Alves Dias ${ }^{2}$

\begin{abstract}
Resumo: O tabagismo compromete a expectativa e a qualidade de vida das pessoas e é considerado um dos mais importantes fatores de risco para a doença periodontal, já que atua na diminuição da resposta imunológica, na vasoconstrição tecidual e alteração da microbiota oral. Diante do exposto este trabalho tem como objetivo descrever, mediante revisão de literatura, os malefícios do tabaco em nível tecidual e celular, suas consequências e prevenção à doença periodontal, além de demonstrar a importância da participação ativa do cirurgião-dentista no processo de orientação por meio de uma abordagem eficaz. Estudos indicam que a terapia periodontal nos fumantes, na maioria das vezes, é insatisfatória em relação aos não fumantes, o que torna muito importante informar e orientar o paciente sobre os riscos e prejuízos no tecido periodontal ao longo do tempo.
\end{abstract}

Palavras-chave: Doença periodontal. Saúde bucal. Tabaco.

\section{The influence of smoking on periodontal disease: A Literature Review}

\begin{abstract}
Smoking compromises people's expectancy and quality of life and is considered one of the most important risk factors for periodontal disease, since it affects the immune response, tissue vasoconstriction and alteration of the oral microbiota. In view of the above, this work aims to describe, through a literature review, tobacco maladies at the tissue and cellular level, its consequences and prevention of periodontal disease, besides demonstrating the importance of the active participation of the dental surgeon in the orientation process by effective approach. Studies indicate that periodontal therapy in smokers, in most cases, is unsatisfactory in relation to nonsmokers, which makes it very important to inform and guide the patient about the risks and damages in the periodontal tissue over time.
\end{abstract}

Keywords: Periodontal disease. Oral health. Tobacco.

\footnotetext{
${ }^{1}$ Graduanda em Odontologia da Faculdade Independente do Nordeste - FAINOR, Vitória da Conquista, Bahia, Brasil. Correspondência: Graziele Veronica Pereira Medeiros (responsável pela correspondência do artigo): Endereço: Avenida Serrinha, número 1054, Bairro Brasil, Vitória da Conquista - BA. CEP: 45051-455, grazielemedeiros93@ gmail.com, telefone: +55 77 9-8809-2416.
}

${ }^{2}$ Mestre em Periodontia, Professora do curso de Odontologia da Faculdade Independente do Nordeste - FAINOR, Vitória da Conquista Bahia, Brasil. Correspondência: Karina Sarno Paes Alves Dias. Endereço: Rua Amélia Moraes, Quadra C, n03, Cond. Morada do bem Querer, Candeias, Vitória da Conquista - BA, Brasil. CEP - 45.028-678, karinasarnopad@ gmail.com, telefone: +55 77 9-9963-9228

470 Id on Line Rev. Mult. Psic. V.12, N. 40. 2018 - ISSN 1981-1179

Edição eletrônica em http://idonline.emnuvens.com.br/id 


\section{Introdução}

Nos últimos anos a necessidade de controle do tabagismo configura um grande desafio da saúde pública, isso porque os fumantes, além de se exporem aos malefícios causados pelo cigarro, ainda atingem as pessoas que os rodeiam e esse hábito gera inúmeras doenças que envolvem quase 2 bilhões de pessoas no mundo (VELSKO et al., 2014). No Brasil, existe uma política pública para o controle do tabagismo desde 1989, no entanto, esse Programa Nacional de Controle do Tabagismo não é suficiente para reduzir o índice de fumantes, mostrando ser importante enfatizar as medidas de prevenção (MOURA et al., 2016).

É notável que o impacto sobre a saúde decorrente do uso do tabaco é latente e, neste contexto, vê-se que o consumo de cigarros está entre um dos principais fatores de risco para câncer de pulmão e muitas outras doenças, a exemplo da periodontite (AL HARTHI et al, 2013). Vale ressaltar que o fumo altera a resposta imune do indivíduo contra os microrganismos periodontopatogênicos, comprometendo assim, o sistema de defesa e podendo gerar a perda de inserção periodontal e alta reabsorção óssea alveolar, elevando, com isso, a probabilidade de perda dentária (CORETTI et al., 2017).

A doença periodontal é uma condição prevalente que pode ser definida como uma inflamação crônica dos tecidos periodontais causada pela infecção bacteriana e advém de um processo de desequilíbrio entre as ações de agressão e defesa sobre os tecidos de sustentação e proteção do dente (LIM; KIM, 2014). Pode ser emergida como gengivite, quando atinge apenas o periodonto de proteção, ou como periodontite, quando ocorre também o comprometimento do periodonto de sustentação (DHADSE; GATTANI; ROHIT, 2010; ARORA, MISHRA, CHUGH 2014).

Peres (2013) destaca que as doenças periodontais têm como sinais clínicos processos inflamatórios de origem infecciosa que acometem os tecidos de proteção (gengivites) e/ou os tecidos de suporte dos dentes (periodontites) gerados por reações inflamatórias e imunológicas nos tecidos periodontais alteradas por microrganismos do biofilme dental, comprometendo o tecido conjuntivo e o osso alveolar.

Logo, é importante acrescer, conforme Arora, Mishra e Chugh (2014), que existe uma correlação positiva entre os níveis salivares de nicotina e a gravidade da doença periodontal, na qual a ação do tabaco no periodonto altera o fluxo sanguíneo gengival, acarreta em uma diferença no número de vasos sanguíneos na gengiva marginal e diminui a resposta 
imunológica, alertando ainda para os efeitos tóxicos da nicotina que geram prejuízo na inserção de fibroblastos à superfície radicular. Além disso, o processo de cicatrização é prejudicado uma vez que o hábito de fumar reduz os níveis séricos de vitamina $\mathrm{C}$, que auxilia na cicatrização (BIZZARRO et al., 2013).

Sendo assim, atuar na prevenção da doença periodontal em pacientes que fumam é necessário, destacando que o melhor método preventivo seria o abandono da prática de fumar, já que o fumo influencia na doença periodontal, agrava sua severidade, incidência e dificulta o seu tratamento (MEULMANN et al., 2013). Por isso, é preponderante que o cirurgião dentista informe e oriente o seu paciente fumante sobre os riscos e os prejuízos no tecido periodontal relacionados ao hábito de fumar (CAMARGO et al., 2016).

\section{Material e Métodos}

Esta pesquisa foi pautada em artigos científicos como base para uma revisão bibliográfica, presente nas bases de dados do Scielo, Pubmed, Medline e Lilacs, com produções publicados entre 2010 e 2017. Para a confecção da revisão, foram localizadas no total, 30 referências (artigos, teses, dissertações) nos quais foram selecionados 21 (vinte e uma) que serviram de base para o presente trabalho.

Foi realizada a busca nos bancos de dados utilizando às terminologias cadastradas nos Descritores em Ciências da Saúde criados pela Biblioteca Virtual em Saúde desenvolvido a partir do Medical Subject Headings da U.S. National Library of Medicine. As palavras-chave utilizadas na busca foram: doença periodontal, saúde bucal e tabaco. A partir daí somente artigos relevantes para a temática em questão e publicados em inglês e/ou português em revistas cientificas foram usados para fazer a discussão dessa temática.

\section{Revisão de Literatura}

O fumo é associado a inúmeras patologias e responsabilizado por altos índices de mortalidade. Estima-se que em todo o mundo, entre os jovens, um em cada cinco é fumante e de 80.000 a 100.000 crianças, começam a fumar todos os dias (MEULMAN et al., 2013). Por 
isso, afirmar que o tabagismo está em ascensão no mundo em desenvolvimento, mas caiu em países desenvolvidos é uma verdade latente, pois o mercado ainda vende cerca de 15 bilhões de cigarros por dia ou 10 milhões a cada minuto (CAMARGO et al., 2016)

O tabagismo causa problemas de saúde, uma vez que no tabaco existem muitas substâncias citotóxicas, como a nicotina, que podem penetrar no tecido mole da cavidade oral, aderir à superfície do dente ou entrar na corrente sanguínea (ARORA; MISHRA; CHUGH, 2014).

O hábito de fumar é um importante fator ambiental associado à destruição periodontal acelerada e, quanto mais for intensificado o seu uso na juventude mais devem ser elaboradas estratégias preventivas para evitar as doenças oriundas desse vício (MONTEIRO et al., 2013).

O tabagismo gera resposta de células inflamatórias e insuficiência de funções celulares, sendo por isso associado a várias doenças, dentre elas a doença periodontal (BERNARDES; FERRES; LOPES, 2013).

Queiroz et al. (2013) e Vettore, Marques e Peres (2013) destacam em seus estudos que a periodontite é uma das doenças crônicas que mais afetam pessoas adultas. Está em segundo lugar nos índices de doenças bucais, perdendo apenas para a cárie dentária, como causa da perda de dentes entre adultos nos países desenvolvidos.

Como o tabagismo está vinculado a um aumento de 2 a 8 vezes da perda óssea e inserção periodontal é relevante frisar que, conforme destaca Coretti et al. (2017), em sua pesquisa comparativa, a má condição de saúde periodontal e a incidência de perda de dentes entre fumantes é muito maior que entre não fumantes.

Sobre os efeitos do tabaco no periodonto, várias análises, como de Peres (2013), Moura et al. (2016), Lim e Kim (2014), Arora, Mishra e Chugh (2014) e Borba et al. (2016) demonstram que fumar é um dos fatores de risco mais importantes e evitáveis na incidência e progressão das doenças periodontais, tendo também efeito negativo sobre todo o espectro de abordagens de tratamento periodontal, como debridamento mecânico, terapia antimicrobiana local e sistêmica, cirurgia periodontal, terapia regenerativa e implantes. Nos últimos 30 a 40 anos, grupos de investigadores desenvolveram várias explicações para a interação complexa de fatores que desempenham um papel na progressão das doenças periodontais e neste contexto os efeitos do tabagismo no periodonto foram amplamente examinados.

Estudos feitos nos EUA por Visvanathan et al. (2014) e Shereef et al. (2015) demonstraram que o tabaco pode ser o fator responsável por metade dos casos de periodontite, 
já que o fumante tem risco 4 vezes maior de ter a doença. Isso porque a nicotina causa alterações celulares que podem gerar o início de uma doença periodontal, comprometendo a imunidade, trazendo efeitos vasoconstritores e citotóxicos e alteração da microbiota patogênica.

Várias pesquisas comprovam que fatores ambientais e comportamentais são significativos para doença periodontal, destacando que o tabagista é mais predisposto a ter doenças bucais e sistêmicas, assim como a progressão da periodontite (SANTOS; SIQUEIRA, 2016).

Dessa forma, enfatizar a questão do tabagismo é preponderante e quando reconhecido como fator de risco ambiental torna-se gerador de doenças, principalmente bucais, como é o caso da periodontite e gengivite. Salienta-se aqui que as doenças periodontais são um fenômeno dinâmico com padrões cíclicos de progressão que podem gerar doenças graves (BORBA et al., 2016).

Muitos estudos, como os de Visvanathan et al. (2014) e Jang, Kwon e Young (2016), têm demonstrado que há uma resposta negativa ao uso do tabaco e este hábito traz o risco de desenvolvimento de câncer bucal, por isso é válido enfatizar que problemas gerados pelo tabagismo, como é o caso das doenças periodontais devem ser prevenidos, pois geralmente ocorrem agravos no quadro, sendo fator de risco latente para doenças que podem levar a morte.

$\mathrm{Na}$ análise feita por Santos e Siqueira (2016) foi demonstrado que o tabagismo causa ruptura e impede a cicatrização dos tecidos periodontais, sendo ainda constatado, de forma frequente, que desempenha um papel significativo no desenvolvimento da periodontite.

A degradação periodontal é considerada uma destruição mais severa entre os fumantes atuais em comparação com os ex-fumantes. E, por esse motivo, o cigarro é tido como um dos fatores de risco mais certos para periodontite, seguido do biofilme dental (SHEREEF et al., 2015; KHAN et al., 2016).

Outras pesquisas como as de Peres (2013) e Arora, Mishra e Chugh (2014) destacaram que o número de cigarros fumados diariamente afeta ainda mais a saúde bucal causando efeitos deletérios sobre o periodonto. Esses estudos demonstram ainda que na periodontite, o tabagismo pode afetar a função dos neutrófilos, impedindo a eliminação de patógenos periodontais e estimulam o dano tecidual mediado pelo estresse oxidativo.

O estudo feito por Jang, Kwon e Young (2016) com grupo de fumantes exibiu uma diminuição em várias citocinas pró-inflamatórias e quimiocinas, além de reguladores de células 
T e células NK. Isso demonstra que os efeitos imunossupressores do tabagismo podem contribuir para uma maior susceptibilidade à periodontite e outras doenças.

Borba et al. (2016) relataram que a nicotina e o hábito de fumar geram na saúde do fumante problemas graves, com mecanismo potencial de perda óssea e maior proliferação de bactérias que são fatores etiológicos na doença periodontal, deixando o paciente mais suscetível à doença. Notaram também que os fumantes têm um número diminuído de linfócitos auxiliares, que são importantes para a função das células B e produção de anticorpos.

O efeito combinado da colonização bacteriana e o efeito local e sistêmico do tabagismo são responsáveis pela maior gravidade da destruição periodontal em fumantes, conforme destaca Jang et al. (2016). Estudos demonstram que, em comparação com os não fumantes, os adultos fumantes apresentam maior prevalência e gravidade da periodontite, além de sangramento gengival e sintomas inflamatórios suprimidos (KHAN et al., 2016).

Há pesquisas que propõem o incentivo e a intensificação das análises epidemiológicas para ajudar na compreensão da epidemiologia da doença periodontal (SHEREEF et al., 2015). Deste modo, seria necessário tratar de forma mais efetiva esse assunto, com pesquisas e análises práticas para oferecer uma estimativa da prevalência do problema periodontal e seus parâmetros clínicos atrelados ao tabagismo (JANG; KWON; YOUNG, 2016).

\section{Discussão}

A periodontite é resultado da interação entre o desafio bacteriano e a resposta imune do hospedeiro, sendo associada a vários fatores predisponentes e modificadores, como fatores dentais locais e estresse, baixo status social, econômico, doenças sistêmicas, álcool e tabagismo (BORBA et al., 2016).

Conforme Jang, Kwon e Young (2016) o cenário atual retrata a relação significativa entre periodontite crônica e frequência de cigarros fumados por dia, ressaltando que o aumento da frequência do tabagismo está associado a um distúrbio na resposta imune celular e humoral e à deterioração da saúde periodontal. Esse achado está de acordo com o estudo de Meulman et al. (2015), Queiroz et al. (2013) e Bizzarro et al. (2013) que relataram fumantes pesados com maior risco de periodontite crônica, em comparação com fumantes moderados ou leves e não fumantes. 
De igual forma, outros autores (VISVANATHAN et al., 2014; SHEREEF et al., 2015; KHAN et al., 2016) demonstraram que o hábito de fumar influencia na doença periodontal, relatando haver uma relação de até 4 vezes mais risco nesses pacientes, devido à alteração da microbiota patogênica, perda óssea e maior proliferação de bactérias. Corroborando com esse achado, Coretti et al. (2017) relataram que o tabagismo está vinculado a um aumento de 2 a 8 vezes da perda óssea e inserção periodontal.

Também em seu trabalho, Bernardes, Ferres, Lopes (2013) demonstraram que a doença periodontal é agravada e até gerada pelo hábito de fumar, assim como Vettore, Marques e Peres (2013) que associaram esse hábito nicotínico ao agravamento e destruição periodontal acelerada.

Trabalhos como o de Peres (2013), Moura et al. (2016), Lim e Kim (2014), Arora, Mishra e Chugh (2014) e Borba et al. (2016) demonstram que fumar é um dos fatores de risco mais importantes e evitáveis na incidência e progressão das doenças periodontais, e é por isso que os efeitos do tabagismo no periodonto são examinados por vários estudos de diversas áreas.

Pesquisas como de Queiroz et al. (2013) demonstraram uma plausibilidade biológica significativa na relação entre tabagismo e doença periodontal. No entanto, relatam que há uma lacuna em relação à dose-resposta do tabagismo e da doença periodontal.

A dependência nicotínica foi avaliada como problema de saúde pública e a OMS tem objetivos distintos para controlar o consumo de tabaco e as taxas de mortalidade em todo o mundo. O impacto do tabagismo é alto na saúde geral e sistêmica. Por isso, autores como Santos e Siqueira (2016), Monteiro et al. (2013) e Camargo et al. (2016) relataram a necessidade de mais políticas públicas para o seu controle, sendo este o principal enfoque de medida preventiva. Al Harthi et al. (2013) reiteraram que o hábito de fumar deve ser combatido por meio da implementação de leis proibitivas de promoção do tabaco, bem como o seu uso em locais públicos.

Foi observado, na maior parte dos trabalhos usados neste estudo, que o tabagismo é um fator potencialmente modificador da doença periodontal, pois ele afeta a resposta imune do hospedeiro, por meio da alteração na função dos neutrófilos, produção de anticorpos, atividades de fibroblastos e fatores vasculares (VISVANATHAN et al., 2014; JANG; KWON; YOUNG, 2016).

Khan et al. (2016) forneceram evidências convincentes sobre a associação entre tabagismo e periodontite crônica na população paquistanesa, com a prevalência de periodontite 
crônica em 81,6\% entre os fumantes. Achados semelhantes foram relatados na população brasileira, nos quais o tabagismo apresentou uma prevalência significativamente maior de periodontite crônica em fumantes, como bem demonstra os estudos de Borba et al. (2016), Monteiro et al. (2013) e Bernardes, Ferres, Lopes (2013).

Observa-se que o tabagismo é considerado um fator de risco potencial para periodontite crônica, pois ele gera a deterioração da saúde periodontal, afetando a qualidade de vida e o bemestar do indivíduo. Dessa forma, é aconselhável que os médicos e profissionais de odontologia eduquem seus pacientes em termos de riscos potenciais do tabagismo para a sua saúde geral e oral (VISVANATHAN et al., 2014).

Jang, Kwon e Young (2016) e Shereef et al. (2015) concluíram que, além disso dos argumentos já mencionados, deve-se fomentar a implementação de programas preventivos e de cessação do tabagismo para melhorar a saúde geral e oral do indivíduo, bem como incentivar mais estudos que proponham a realização de pesquisas epidemiológicas que possam ajudar na compreensão da epidemiologia da doença periodontal no mundo.

\section{Conclusão}

Conforme estudos, observa-se que existe uma plausibilidade biológica significativa na relação entre tabagismo e doença periodontal com influência da primeira sobre a segunda, salientando que o fumo favorece, em pessoas com periodontite, um agravamento na perda de inserção e perda óssea alveolar, podendo acarretar a perda dentária. Sendo assim, é possível afirmar que em fumantes a doença periodontal apresenta uma maior gravidade. Nesse contexto, é necessário a atuação do cirurgião dentista quanto a informar aos seus pacientes dos malefícios do cigarro para a saúde bucal e o quão importante é ter hábito de higiene oral, prevenindo assim doenças na cavidade oral.

\section{Referências}

AL HARTHI, L.S. et al. The impact of periodontitis on oral health-related quality of life: A review of the evidence from observational studies. Aust Dent, v. 58, n. 384, p. 274-7, 2013. 
ARORA, N; MISHRA, A; CHUGH, S. Microbial role in periodontitis: Have we reached the top? Some unsung bacteria other than red complex. J Indian Soc Periodontol, v. 18, n. 3, p. 9-13, 2014.

BERNARDES, V.; FERRES, M. O; LOPES, J. W. O tabagismo e as doenças periodontais. Faculdade de Odontologia de Lins/Unimep, v. 23, n. 1, p. 37-45, 2013.

BIZZARRO, S. et al. Subgingival microbiome in smokers and non-smokers in periodontitis: An exploratory study using traditional targeted techniques and a next-generation sequencing. $J$ Clin Periodontol, v. 40, n.4, p. 483-92, 2013.

BORBA, T.T. et al. Associação entre periodontite e fatores sociodemográficos, índice de massa corporal e características do estilo de vida. Revista de Epidemiologia e Controle de Infecção, Santa Cruz do Sul, v. 6, n. 4, p. 2238-3360, 2016.

CAMARGO, G.A. et al. Aspectos clínicos, microbiológicos e tratamento periodontal em pacientes fumantes portadores de doença periodontal crônica: revisão de literatura. Rev. bras. odontol., Rio de Janeiro, v. 73, n. 4, p. 325-30, 2016.

CORETTI, L. et al. Subgingival dysbiosis in smoker and non-smoker patients with chronic periodontitis. Molecular Medicine Reports, 2017; 15 (1): 2; 07-14.

DHADSE, P. GATTANI, D. ROHIT, M. The link between periodontal disease and cardiovascular disease: How far we have come in last two decades? J Indian Soc Periodontol, v. 14, n. 3, p.148-54, 2010.

JANG, Y.; KWON; J. YOUNG, J. Association between Smoking and Periodontal Disease in Korean Adults: The Fifth Korea National Health and Nutrition Examination Survey (2010 and 2012). Korean J Fam Med, v. 37, n. 2, p. 117-122, 2016.

KHAN, S. et al. Chronic periodontitis and smoking Prevalence and dose-response relationship. Saudi Med J. v. 37, n. 8, p. 889-894, 2016.

LIM, H.C. KIM, C.S. Oral signs of acute leukemia for early detection. J Periodontal Implant Sci. v. 44, n. 2, p. 293-9, 2014.

MEULMAN, T. et al. One stage, full-mouth, ultrasonic debridement in the treatment of severe chronic periodontitis in smokers: a preliminary, blind and randomized clinical trial. J Int Acad Periodontol, v. 15, n. 3, p. 83-90, 2013.

MONTEIRO, F. et al. Characterization of the oral fungal microbiota in smokers and nonsmokers. Eur J Oral Sci. v. 121, n. 2, p. 132-5, 2013.

MOURA, S. K. et al. Influência do Hábito de Fumar na Prevalência da Doença Periodontal em Idosos do Município de Londrina, Pruniciências, v. 20, n. 1, p. 62-7, 2016. 
PERES, M. A. Perdas dentárias no Brasil: análise da Pesquisa Nacional de Saúde Bucal 2010. Rev. Saúde Publica, v. 47, n. 4, p. 78-89, 2013.

QUEIROZ, A. et al. Adjunctive effect of antimicrobial photodynamic therapy to nonsurgical periodontal treatment in smokers: a randomized clinical trial. Lasers Med Sci. v. 30, n. 2, p. 1379-81, 2013.

SANTOS, V; SIQUEIRA, L. C. Tabaco e Doenças Periodontais. Rev. Cient. In FOC, v. 1, n. 1, p. 89-98, 2016.

SHEREEF, M. et al. The effect of cigarette smoking on the severity of periodontal diseases among adults of Kothamangalam Town, Kerala. J Pharm Bioallied Sci. v. 7, n. 2, p. 648-651, 2015 .

VELSKO, I. et al. Active invasion of oral and aortic tissues by porphyromonas gingivalis in mice causally links periodontitis and atherosclerosis. Plos One, v. 9, n. 1, p. 97-111, 2014.

VETTORE, M. MARQUES, R.A, PERES, M. A. Desigualdades sociais e doença periodontal no estudo SBBrasil 2010: abordagem multinível. Rev. Saúde Pública, v. 47, n. 3, p. 29-39, 2013.

VISVANATHAN, R. et al. Effect of Smoking on Periodontal Health. J Clin Diagn Res, v. 8, n. 7, p. 46-49, 2014.

\section{Como citar este artigo (Formato ABNT):}

MEDEIROS, Graziele Veronica P.; DIAS, Karina Sarno Paes Alves. A influência do tabagismo na doença periodontal: Uma Revisão de Literatura. Id on Line Rev.Mult. Psic., 2018, vol.12, n.40, p.470-479. ISSN: 1981-1179.

Recebido: 16/04/2018

Aceito 18/04/2018 